\title{
The Effect of Shadow Lines on a Low Concentrating Photovoltaic System
}

\author{
H.J.J. Janssen, P.J. Sonneveld, G.L.A.M. Swinkels, B.A.J. van Tuijl and \\ H.F. de Zwart \\ Wageningen UR Greenhouse Technology, P.O. Box 644, 6700 AA Wageningen, tel: +31317483329 fax: \\ +31317418094,e-mail: hansjj.janssen@wur.nl, internet: www.greenhousehorticulture.wur.nl
}

\begin{abstract}
In order to reduce the energy losses caused by shadow lines, three options are investigated. These are: 1 . the use of two types of diodes; 2. the use of an "ideal" diode based an active bypass by using MOS-FET"s [4] and 3. parallel switching of a number of cells between two shadow lines. The first method can reduce the voltage losses of the diode to about $300 \mathrm{mV}$ when oversized Schottky diodes are used. With the second method it is possible to reduce the voltage losses further to about $60 \mathrm{mV}$ for FETs with a resistance of $3 \mathrm{~m} \Omega$. This method has as disadvantage that more electronic components are required to control the FET. With the third method about 10 cells are placed in parallel in one module. In that case only one shadow line appears on each module. Series connection of these parallel modules will result in zero energy losses because no bypass diodes are needed at all. This method has as a disadvantage of very high current output of the module of up to 200A. In a model, the three methods are further analyzed with respect of power losses and costs. The effect of the degree of shadowing on the daily energy yield and hence the fill factor is simulated with a model of the PV module. These results can be used in the design of the circuit of the ideal bypass diode.
\end{abstract}

Keywords: Greenhouse, Fresnel lens, Solar Concentrator, Silicon Solar Cells, Active Bypass

PACS: 88.40.jj, 88.40.jm, 42.79.Ek, 85.30.Hi, 85.30.Kk

\section{INTRODUCTION}

During measurements on an low concentrating Photovoltaic system (LCPV) based on reflective mirrors [3] it was found that shadow lines appear due to the low reflection of the glass rods of the construction of the mirror. This mirror was integrated in the construction of a building, a greenhouse. Without provisions, these shadow lines on the CPV module (Figure 1) will result in considerable drop in the energy yield of the system. The power measurements on the system show a very low fill factor less of 0.1. In another LCPV system based on a Fresnel lens system the effect of the shadow lines was diminished by ordinary parallel bypass diodes on each cell. This second system reveals an increased fill factor of about 0.22 . Despite of this increase, the fill factor was too low for performing the system in practice. The result of a shadow line is still twofold: 1 . the module voltage drops with about $0.6 \mathrm{~V}$, which is the voltage of one cell, and 2. the module voltage drop is again $0.6 \mathrm{~V}$, due to the bypass diode losses. Therefore the energy loss at a current of $20 \mathrm{~A}$ will be $24 \mathrm{~W}$ per shadowed PV cell. In this investigation different options are studied to diminish these energy losses.

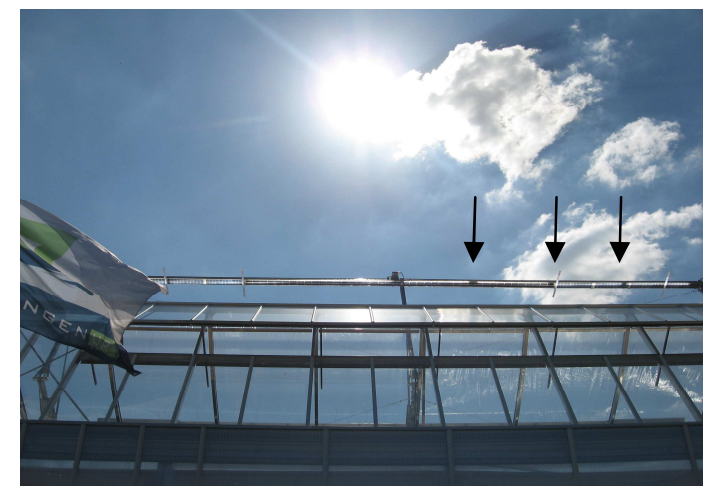

FIGURE 1. The occurrence of shadow lines on the solar collector. 


\section{MATERIALS AND METHODS}

\section{C \\ FIGURE 2 Schottky bypass diodes for each CPV-cell}

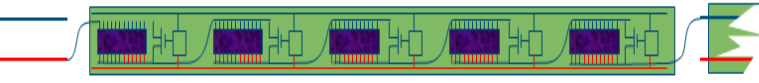

FIGURE 3 Ideal bypass diodes implemented as Switches with MOS-FET's as active bypass for each CPV-cell

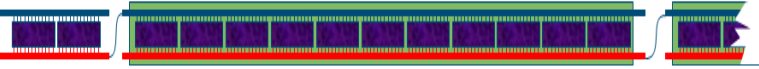 \\ FIGURE 4 Special parallel-series connection: each "unit length" of the distance between glass rods is parallel connected and then in series.}

In order to reduce the energy losses caused by shadow lines, three options are investigated. Option 1: the use of better (Schottky) diodes (see Figure 2); Option 2: the use of an "Ideal" Diode Module (IDM) based on MOS-FET's forming an active bypass (Figure 3 ). The effect and mathematical modeling of different bypass circuits is described by B. Prasad et all [4]. Option 3: parallel switching of a number of cells between two shadow lines (Figure 4).

The first method can reduce the voltage losses of the diode to about $300 \mathrm{mV}$ when oversized Schottky diodes are used. With the second method it is possible to reduce the voltage losses further to about $60 \mathrm{mV}$ for FET's with a resistance of $3 \mathrm{~m} \Omega$. This method has as disadvantage that more electronic components are required to control the FET. If solution 1 and 2 are combined, a malfunction of the IDM will be partially compensated by the Schottky bypass, unless the IDM forms a short-circuit. In general the reliability reduces with the number of components. With the third method, a number of cells with the same total length as the hart-to-hart distance between the glass rods (normally about 10 cells) are placed in parallel in one module. In that case only one shadow line appears on each module. Series connection of these parallel modules will result in zero energy losses because no bypass diodes are needed at all. This method has as a disadvantage of very high current output of the module of up to 200A and low output voltages. The high currents need massive and costly cabling and the low voltages result in low efficiency at the transformation to the grid. In a model, the three methods are further analyzed with respect of power losses and costs. The effect of the degree of shadowing on the daily energy yield and hence the fill factor is simulated with a model of the bypass diode. These results can be used in the design of the circuit of the ideal bypass diode.

\section{RESULTS}

\section{The simulations}

In the simulation, the shadow line moves only over two PV cells of a module of 10 cells (Figure 5a. The shade, having a width equal to the size of the cell, changes linear in time and as a result the generated current $\mathrm{I}_{\mathrm{PV}}$ will decreases from $25 \mathrm{~A}$ to $0 \mathrm{~A}$ and back again. During shading, the bypass takes over and the load power $\mathrm{P}_{\mathrm{Load}}$ of the total PV module will decrease (Figure 5b). Also the voltage $\mathrm{U}_{\mathrm{PV} 1}$ and $\mathrm{U}_{\mathrm{PV} 2}$ over the PV cell gets negative (Figure 5e,f). In the simulation the load resistance is variable and keeps the load constantly at the Maximum Power Point. This simulation shows that the Ideal Diode Module IDM (solid graphs) performs much better than the Schottky diode (dashed graphs). The normal diode (dotted graphs) performs very bad especially when two cells are half shaded (at $\mathrm{t}=5$ ).

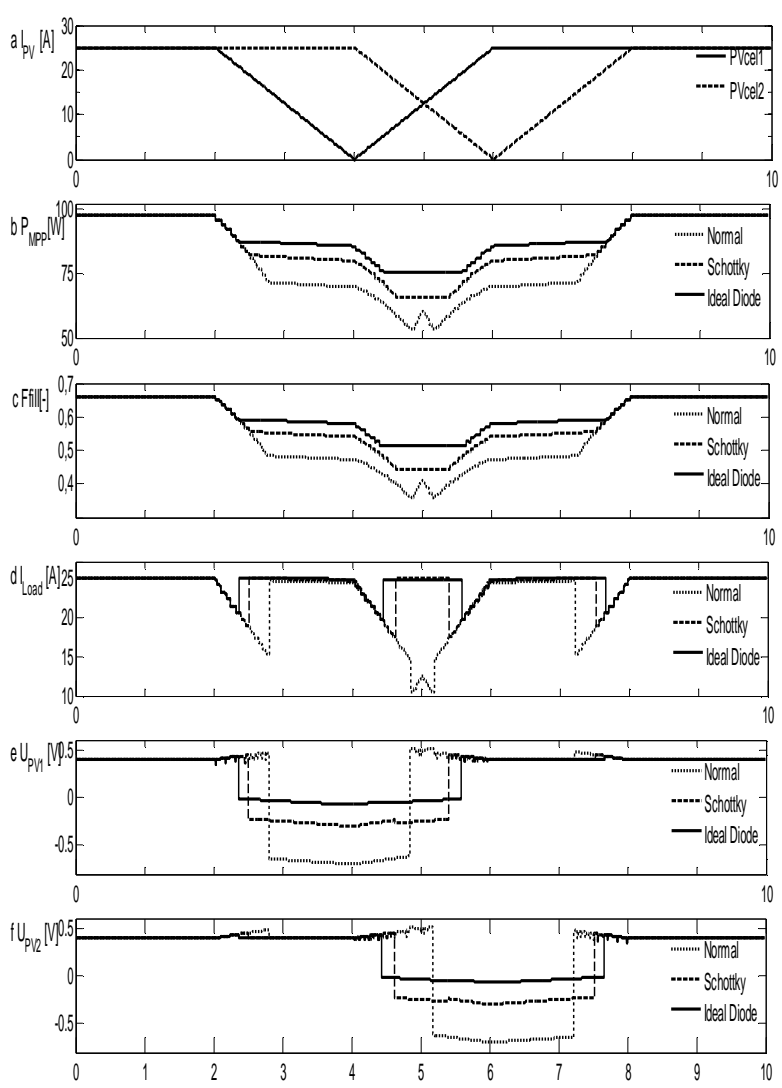

Figure 5. Simulation of a moving shadow line over a PV module. 
The simulation shows a power load $\mathrm{P}_{\text {Load }}=97.5 \mathrm{~W}$ at unshaded condition. (Figure $5 \mathrm{~b}$ ) At $\mathrm{t}=5$ the power loss with the application of a bypass of type IDM is $21.5 \mathrm{~W}$,of type Schottky is $31.2 \mathrm{~W}$ and for the normal diode is $42 \mathrm{~W}$. In the simulation all parameters of the PV cell, bypass diodes and MOS-FET's the different threshold voltages and resistances are taken in account. As expected, the simulation shows that the total efficiency, and hence the fill factor, is very sensitive for these parameters. Simulation with the IDM option shows a fill factor of 0.65 at no shade condition and a fill factor of 0.55 at a condition of one shadow line.

\section{The test PV cell.}

In the project with the reflecting mirror(see Figure 1), a test PV cell was used to overcome the influence of shadow. In the latest project [1,2] a test PV cell is used to study the characteristics of the cell and the influence of the shadow lines. The measured $\mathrm{V}_{\mathrm{OC}}$ is $0.56 \mathrm{~V}$, the $\mathrm{I}_{\mathrm{SC}}$ reaches values up to $25 \mathrm{~A}$, the internal resistance $R_{s}$ is $10 \mathrm{~m} \Omega$. The short circuit current is measured with a current sense resistor of $1 \mathrm{~m} \Omega$ in parallel with the PV cell. This result is used in the simulations.

\section{The Ideal Diode Module (Active Bypass).}

In general, a single PV cell should be switched on only if it contributes to the total power of the PV module. A power management per group of PV modules could achieve this but requires communication/control of every active bypass. Figure $5 \mathrm{~b}, \mathrm{~d}$ shows the relation between the power and the main current. If the voltage over the combination PV cell/bypass is positive the cell produces power, if the voltage is negative the bypass absorbs energy and should be kept low. The current generation of the cell is proportional to the irradiation. Shade reduces this current and, as a result of the series connection, also reduces the main current. Switching on and off with very low difference in level (hysteresis) makes the system instable (oscillating). A small delay in time between switching on/off improves the stability. In this project a special IDM is developed, a pico processor controls 4 MOS-FET switches. The level on which the switches are switched on/off and the time delay are all programmable. By combining 4 active bypass circuits. there is always at least $2 * 0.6 \mathrm{~V}$ available for power supply of the electronics ( at maximum two of the four cells are shaded). As soon as these parameters are known from practice, a simpler IDM based on basic analog circuitry can be developed and the costs can be reduced. On the other hand, the digital version offers optimal (central) power management hence lowest losses. The cost of the experimental combined four IDM's is about $€ 12$,- on components where the main costs are the MOS-FET's and bypass diodes. In principle the bypass diodes are not needed but are a backup if the MOS-FET switches fail, one bypass diode on four switches is also an option, so it is costs versus reliability and losses.

\section{Comparisons on cost and performance.}

The size and number of the PV cells was one of the design criteria, so more small cells vs. less large cells. Between the cells a minimal gap is needed for connecting the cells. The total area of these gaps increases with the number of cells, hence the losses increase because this area is inactive. At a low number of cells the permanent shadow lines of the greenhouse construction now causes large cells to be bypassed, also an increase in losses.

$$
\text { Costs }[€] \quad \text { Current }[\mathrm{A}]
$$

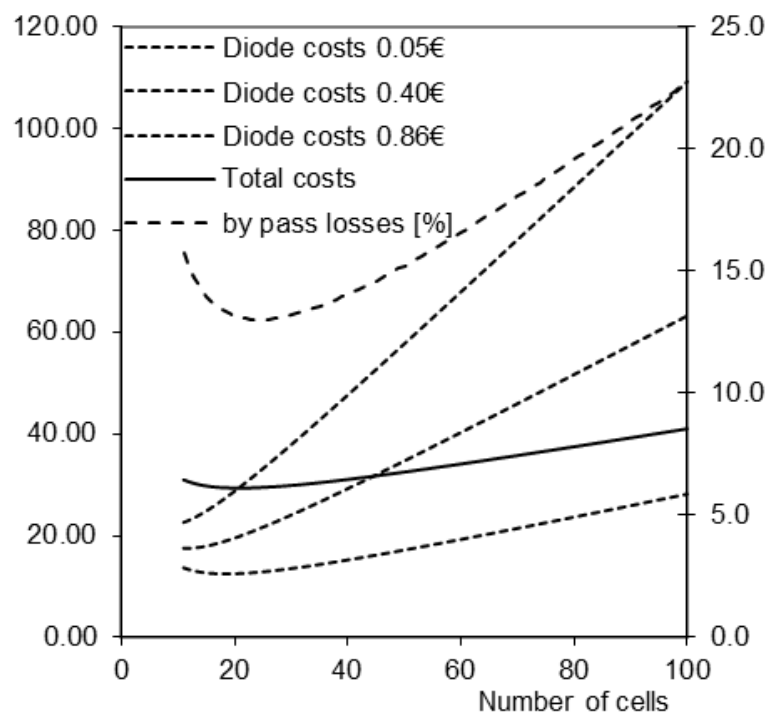

Figure 6 Comparison of the three methods.

The solid curve in Figure 6 represents total costs as a function of the number of cells of the system with bypass diodes. The dashed curve in Figure 6 represents the extra losses of the bypass diodes compared with parallel-series connection. The tradeoff is around a number of 20 cells per hart-to-hart distance of the shadow lines. At a large number of small cells (low current) a large number of small (cheaper) bypass diodes is needed. At a small number of cells (high current) heavy (expensive) bypass diodes are needed. In Figure 6 the dotted curves represent the costs of the heavy, medium and small bypass diodes. A large number of cells require more labor. The total costs (Figure 6 solid curve) is the result of a combination of the three types of bypass diodes and the costs of labor. The IDM is still experimental and not yet taken into account but it is of the type heavy bypass diode and the cost of components is at least $€ 3$. 


\section{CONCLUSIONS}

To overcome the drawback of shadow lines, the application of the special parallel-series connection has the highest efficiency and needs no extra components but is hardly practical due to extreme high currents and low output voltages. The series connection of PV cells requires bypass circuits. Normal diodes as bypass result in losses too high and are not practical. The application of Schottky bypass diodes is practical, economical and has medium losses. The application with "ideal" bypass diodes gives an improved efficiency (fill factor) but requires more components, hence higher costs and lower reliability. Not all the results are yet available but it is clear that all resistances in the circuit like wiring, soldering joints, connectors etc. are of great influence on the overall efficiency. Great care should be taken to minimize all these resistances in the design and construction of the PV modules.

\section{ACKNOWLEDGMENTS}

This research is financially supported by the Dutch Product Board for Horticulture and Ministry of Economic Affairs, Agriculture and Innovations The Netherlands.

\section{REFERENCES}

1. P.J. Sonneveld, G.L.A.M. Swinkels, B.A.J .van Tuijl, H. Janssen, J.H. van Campen, G.P.A. Bot, Energy yield of a CPV System with Static Linear Fresnel Lenses integrated in a Greenhouse, Solar Energy, 85, 432-442 (2011)

2. P.J. Sonneveld, G.L.A.M. Swinkels, B.A.J. van Tuijl, H.J.J. Janssen, H.F. de Zwart, Static Linear Fresnel Lenses as LCPV System in a Greenhouse CPV-7 2011

3. P.J. Sonneveld, G.L.A.M. Swinkels, J. van Campen, B.A.J van Tuijl, H.J.J. Janssen, G.P.A. Bot (2010) Measurements on a solar greenhouse combining cooling and electrical energy production, Biosystems Engineering, 106, p.48-57

4. B. Prasad Koirala, B. Sahan, N. Henze, Study on MPP Mismatch Losses in Photovoltaic Applications, 24th European Photovoltaic Solar Energy Conference and Exhibition, Hamburg/Germany, 21.-25. September 2009 